\title{
An efficient multi-time step FEM-SFEM iterative coupling procedure for elastic-acoustic interaction problems
}

\author{
J.E.A. Silva - F.S. Loureiro - W.J. \\ Mansur • L.C. Wrobel
}

Received: date / Accepted: date

\begin{abstract}
An iterative coupling methodology between the Finite Element Method (FEM) and the Spectral Finite Element Method (SFEM) for the modeling of coupled elastic-acoustic problems in the time domain is presented here. Since the iterative coupling procedure allows the use of a nonconforming mesh at the interface between the subdomains, the difference in the element sizes concerning the FEM and SFEM is handled in a straightforward and efficient manner, thereby retaining all the advantages of the SFEM. By means of the HHT time integration method, controllable numerical damping can be introduced in one of the subdomains, increasing the robustness of the method and improving the accuracy of the results; besides, independent time-step sizes can be considered within each subdomain, resulting in a more efficient algorithm. In this work, a modification in the subcycling procedure is proposed, ensuring not only an efficient and accurate methodology but also avoiding the computation of a relaxation parameter. Numerical simulations are presented in order to illustrate the accuracy and potential of the proposed methodology.
\end{abstract}

Keywords Spectral finite element · Acoustic fluid · Elastodynamics · Multi-time stepping scheme $\cdot$ Iterative coupling

J.E.A. Silva

Postgraduate Program in Computational Modeling, Federal University of Juiz de Fora, Brazil

E-mail: jeas560@gmail.com

F.S. Loureiro

Department of Thermal and Fluid Sciences, Federal University of São João del-Rei, Brazil

W.J. Mansur

Department of Civil Engineering, COPPE/Federal University of Rio de Janeiro, Brazil

L.C. Wrobel

Department of Mechanical, Aerospace and Civil Engineering, Brunel University London, England 


\section{Introduction}

In the seventies, many works were carried out to develop numerical schemes for the solution of coupled systems such as solid-fluid and soil-structure. For instance, one can quote the node-per-node partitioning $[3,4]$ and the elementper-element partitioning algorithms [20-22], adopting the FEM for both subdomains and allowing the use of different time integration techniques (i.e., implicit-explicit). On the other hand, the application and theoretical studies of different spatial discretization schemes able to better represent the distinct features of each subdomain have emerged as an important topic of research.

Concerning the solution process of coupled systems, one can generally classify it as direct or iterative. In the direct coupling methodology (that is the case of the aforementioned cited papers), a large block-matrix system of equations is obtained after taking into account a suitable discretization procedure of the two subdomains simultaneously. In this way, the following main drawbacks may arise: i) ill-conditioning of the global coupled matrix, especially when the subdomains present very different physical properties or whether different spatial discretization techniques are employed for each subdomain; ii) increase in the memory storage and computational time to solve the full system, since the use of preconditioned iterative solvers is not straightforward; and iii) difficulty in establishing a straightforward formulation for coupling two distinct spatial and/or time discretization schemes. Another issue of great importance concerns the formulations to deal with non-matching nodes at the coupling interface (i.e., nonconforming mesh). One widely employed formulation is based on the use of Lagrange multipliers $[5,40]$ which adds other unknown variables to the problem solution and may increase the ill-conditioning of the final matrix system, making the use of iterative solvers a great challenge.

On the other hand, in a great deal of cases, iterative coupling methodologies seem to be an attractive alternative to the direct ones in the sense that each subdomain is analyzed separately and only the information on the coupling interface needs to be transfered through the subdomains [27,12]. Hence, a number of advantages can be highlighted, namely: i) it is possible to use a specific discretization technique and solver that fit better for each subdomain, since they are analyzed separately, improving the effectiveness of the simulations; ii) the non-matching nodes at the coupling interface (nonconforming mesh) are readily handled by means of a simple interpolation procedure; and iii) an interpolation and/or extrapolation procedure can also be adopted in the time-marching process to deal with different time-step sizes used in the subdomains. In spite of all these advantages and a straightforward computational implementation, the major drawback of the iterative coupling methodologies stems from the lack of a rigorous general mathematical convergence analysis. Another of the major issues of the iterative coupling procedure is concerned with the number of iterations required for convergence. It is well-known that the introduction of a relaxation parameter with or without adaptive strategies for the relevant variables at the coupling interface may considerably improve the convergence $[27,11,13]$. 
It is well-known that the SFEM has been firstly proposed to solve fluid dynamic problems by Patera [31]. Since then, the method has also been successfully applied to other kind of problems [29,8]. For instance, the SFEM has been widely employed in wave propagation problems for both acoustic and elastic media $[26,23,17]$ due to its high accuracy with few nodes per wavelength compared to the standard FEM, resulting in a lower memory storage requirement for large-scale problems. Furthermore, in a different way of the traditional high-order FEM formulation, the interpolation functions regarding the SFEM do not present the Runge phenomenon (i.e, spurious oscillations) [6], therefore yielding a more robust formulation. However, in some cases, the presence of highly distorted spectral elements in the mesh may deteriorate the quality of the solution $[32,25]$.

In the scope of the FEM-SFEM coupling, one can mention some works using Lagrange multipliers and mortar methods [9,30]. However, to the best of authors' knowledge, this is the first study in the direction of applying an iterative coupling methodology between these two numerical methods for the solution of solid-fluid time-domain problems. The assumption of acoustic fluid is assumed herein simplifying the solid-fluid model [2]. Therefore, approximating the acoustic fluid by the SFEM and the solid by the FEM and owing to the inherent nonconforming mesh at the coupling interface, an efficient numerical approach is established.

Although in some works such as $[36,15,10,16]$ adaptive relaxation parameters for dynamic problems have been proposed and a good improvement has been attained, here a strategy without the use of relaxation parameters is developed in an attempt to improve even more the efficiency of the iterative coupling process. This is accomplished by performing a modification in the subcycling procedure, achieving performances equal or even better than those procedures with relaxation parameters.

The paper is organized as follows. First, a brief review of both the FEM and SFEM discretization methods for a solid-fluid coupled model and the time-marching scheme implemented for this work are presented. In the sequence, the proposed multi-time step iterative coupling technique between the FEM-SFEM methods is described with a appropriate mathematical rigor. At the end of the paper, two numerical examples are presented illustrating the effectiveness, accuracy and flexibility of the proposed methodology.

\section{FEM-SFEM coupling for solid-acoustic transient problems}

\subsection{FEM for solids with coupling interface}

Let $\Omega_{s} \subset \mathbb{R}^{d}$ be a bounded domain, where $d$ is the number of spatial dimensions of the problem under consideration with $s$ being related with the solid subdomain, and let $I=(0, T] \subset \mathbb{R}^{+}$be the time domain of the analysis. Elastodynamic problems are mathematically modeled by the following set of 
equations:

$$
\rho_{s} \ddot{u}_{i}^{s}-\sigma_{i j, j}=b_{i}^{s} \text { in } \Omega_{s} \times I
$$

where $u_{i}^{s}: \Omega_{s} \times I \rightarrow \mathbb{R}, b_{i}^{s}: \Omega_{s} \times I \rightarrow \mathbb{R}$ and $\sigma_{i j}: \Omega_{s} \times I \rightarrow \mathbb{R}$ stand, respectively, for the displacements, given body force per unit volume and Cauchy stress tensor components; and $\rho_{s}: \Omega_{s} \rightarrow \mathbb{R}^{+}$is the mass density.

Moreover, considering the boundary partition $\partial \Omega_{s}=\Gamma_{s}=\Gamma_{D_{i}} \cup \Gamma_{N_{i}} \cup \Gamma_{T_{i}}$ with $\Gamma_{D_{i}} \cap \Gamma_{N_{i}}=\Gamma_{D_{i}} \cap \Gamma_{T_{i}}=\Gamma_{N_{i}} \cap \Gamma_{T_{i}}=\emptyset$, where $\Gamma_{T_{i}}$ is the surface relating to the coupling interface, the boundary conditions are given by:

$$
u_{i}^{s}=\bar{u}_{i}^{s} \text { on } \Gamma_{D_{i}} \times I, \quad \tau_{i} \equiv \sigma_{i j} n_{j}^{s}=\bar{\tau}_{i} \text { on } \Gamma_{N_{i}} \times I, \quad \tau_{i}=-p n_{i}^{s} \text { on } \Gamma_{T_{i}} \times I
$$

where $\bar{u}_{i}^{s}: \Gamma_{D_{i}} \times I \rightarrow \mathbb{R}$ are prescribed displacements and $\bar{\tau}_{i}: \Gamma_{N_{i}} \times I \rightarrow \mathbb{R}$ are prescribed tractions with $n_{j}^{s}$ being the outward normal vector components on $\Gamma_{T_{i}}, p: \Gamma_{T_{i}} \times I \rightarrow \mathbb{R}$ stands for the hydrodynamic pressure at the coupling interface from the fluid subdomain to be properly defined later on.

To derive the variational form of the problem described by the above equations, we start by defining the space of admissible solutions, where the solution $u_{i}^{s}$ is sought to be in $\mathbf{S}=\left\{u_{i}^{s} \mid u_{i}^{s}(\cdot, t) \in H^{1}\left(\Omega_{s}\right), u_{i}=\bar{u}_{i}\right.$ on $\left.\Gamma_{D_{i}} \times I, i=1, \ldots, d\right\}$ and the space $\mathbf{V}$ of the test functions $w_{i}$ is defined as $\mathbf{V}=\left\{w_{i} \mid w_{i} \in H^{1}\left(\Omega_{s}\right), w_{i}=0\right.$ on $\left.\Gamma_{D_{i}}, i=1, \ldots, d\right\}$, where $H^{1}$ is the classical Sobolev space that denotes the space of square-integrable functions with square-integrable generalized first derivatives [1]. Thus, the variational form of the problem under consideration can be written as: find $\mathbf{u}^{s} \in \mathbf{S}$, such that $\forall t>0$ and $\forall \mathbf{w} \in \mathbf{V}[19]$

$$
\left(w_{i}, \rho \ddot{u}_{i}^{s}\right)+a_{s}\left(w_{i}, u_{i}^{s}\right)=\left(w_{i}, b_{i}^{s}\right)+\left(w_{i}, \bar{\tau}_{i}\right)_{\Gamma_{N_{i}}}+\left(w_{i}, \tau_{i}\right)_{\Gamma_{T_{i}}}
$$

where $(\cdot, \cdot) \equiv(\cdot, \cdot)_{\Omega_{s}}$ and $(\cdot, \cdot)_{\Gamma}$ is the classical $L^{2}$-inner product in $\Omega_{s}$ and on $\Gamma$, respectively, and the $H^{1}$ bilinear form $a_{s}(\cdot, \cdot)$ is defined as $a_{s}\left(w_{i}, u_{i}^{s}\right)=$ $\int_{\Omega_{s}} C_{i j k l} u_{k, l}^{s} w_{i, j} d \Omega_{s}$, with $C_{i j k l}: \Omega_{s} \rightarrow \mathbb{R}$ being the material elasticity tensor components, in this work considered in a linear and isotropic regime (i.e. $\sigma_{i j}=$ $\left.C_{i j k l} u_{k, l}^{s}\right)$.

Then, by means of the semi-discrete FEM formulation where the spatial domain is discretized independently of the time domain, the following ODE system is obtained [2]:

$$
\mathbf{M}_{s} \ddot{\mathbf{U}}+\mathbf{K}_{s} \mathbf{U}=\mathbf{F}_{s}+\mathbf{F}_{T}
$$

where $\mathbf{M}_{s} \in \mathbb{R}^{n q_{s} \times n q_{s}}$ and $\mathbf{K}_{s} \in \mathbb{R}^{n q_{s} \times n q_{s}}$ denote the standard mass and stiffness matrices respectively, the vectors $\mathbf{U}: I \rightarrow \mathbb{R}^{n q_{s}}, \ddot{\mathbf{U}}: I \rightarrow \mathbb{R}^{n q_{s}}, \mathbf{F}_{s}$ : $I \rightarrow \mathbb{R}^{n q_{s}}$ and $\mathbf{F}_{T}: I \rightarrow \mathbb{R}^{n q_{s}}$ represent, respectively, the nodal displacements, accelerations, external forces and coupling forces acting on the solid subdomain due to the interaction of the fluid subdomain, with $n q_{s}$ being the number of equations in the solid model. The numerical treatment of $\mathbf{F}_{T}$ is presented in section 3 . 
2.2 SFEM modeling for acoustic fluids with coupling interface

In order to obtain the description of an acoustic fluid, the Navier-Stokes equations are simplified under the following assumptions: i) negligible advective terms; ii) inviscid (negligible viscous terms) and compressible (with small variations for the density) flow; and iii) small displacements for the fluid particles. Thus, the momentum and mass conservation equations can be written as [39]:

$$
\begin{array}{r}
\rho_{f} \ddot{u}_{i}^{f}+p_{, i}-f_{i}^{f}=0 \text { in } \Omega_{f} \times I \\
\dot{u}_{i, i}^{f}+\dot{p} / \kappa_{f}=0 \text { in } \Omega_{f} \times I
\end{array}
$$

where $p: \Omega_{f} \times I \rightarrow \mathbb{R}$ represents the hydrodynamic pressure field, $\dot{u}_{i}^{f}: \Omega_{f} \times I \rightarrow$ $\mathbb{R}$ and $\ddot{u}_{i}^{f}: \Omega_{f} \times I \rightarrow \mathbb{R}$ the fluid velocities and accelerations fields, respectively, $f_{i}^{f}: \Omega_{f} \times I \rightarrow \mathbb{R}$ the volume forces, $\rho_{f}: \Omega_{f} \rightarrow \mathbb{R}_{+}$and $\kappa_{f}: \Omega_{f} \rightarrow \mathbb{R}_{+}$the density and compressibility coefficient, respectively; all with respect to the fluid subdomain $\Omega_{f}$.

By rewriting Eq. (5) in terms of the pressure only, one obtains the following equation that models acoustic fluids [2]:

$$
p_{, i i}-\frac{1}{v_{f}^{2}} \ddot{p}+\frac{b^{f}}{\kappa_{f}}=0 \text { in } \Omega_{f} \times I
$$

where $v_{f}=\sqrt{\kappa_{f} / \rho_{f}}$ is the acoustic wave velocity and $b^{f}: \Omega_{f} \times I \rightarrow \mathbb{R}$ represents the source in the fluid. Although this is a simplified model it is often adopted in many simulation problems.

In addition to the standard boundary conditions, the usual absorbing and coupling boundary conditions are also employed, yielding the boundary partition $\partial \Omega_{f}=\Gamma_{f}=\Gamma_{1} \cup \Gamma_{2} \cup \Gamma_{3} \cup \Gamma_{4}$ and $\Gamma_{i} \cap \Gamma_{j}=\emptyset$ when $i \neq j$, in which:

$$
\begin{array}{r}
p=\bar{p} \text { on } \Gamma_{1} \times I ; \quad q \equiv p_{, j} n_{j}^{f}=\bar{q} \text { on } \Gamma_{2} \times I \\
q=-\frac{1}{v_{f}} \dot{p} \text { on } \Gamma_{3} \times I ; \quad q=\rho_{f} \ddot{u}_{i}^{s} n_{i}^{s} \text { on } \Gamma_{4} \times I
\end{array}
$$

where $\bar{p}: \Gamma_{1} \times I \rightarrow \mathbb{R}$ is the prescribed pressure, $\bar{q}: \Gamma_{2} \times I \rightarrow \mathbb{R}$ is the so-called prescribed surface flux, $\ddot{u}_{i}^{s}$ and $n_{i}^{s}$ stand for accelerations and the unit outward normal vector components from $\Omega_{s}$ (solid subdomain).

In a similar way of that in the solid model, the weak form of the fluid model consists in finding $p \in S_{f}$, such that $\forall t>0$ and $\forall w \in V_{f}$ :

$$
\left(w, \frac{1}{v_{f}^{2}} \ddot{p}\right)+a_{f}(w, p)=\left(w, \frac{1}{\kappa_{f}} b^{f}\right)+(w, \bar{q})_{\Gamma_{2}}+\left(w, \frac{1}{v_{f}} \dot{p}\right)_{\Gamma_{3}}+(w, q)_{\Gamma_{4}}
$$

where $(\cdot, \cdot) \equiv(\cdot, \cdot)_{\Omega_{f}},(\cdot, \cdot)_{\Gamma_{i}}$ with $i=1, \ldots, 4$, and $a_{f}(w, p)=\int_{\Omega_{f}} w_{, i} p_{, i} d \Omega_{f}$ in which $\quad S_{f}=\left\{p \mid p(\cdot, t) \in H^{1}\left(\Omega_{f}\right), p=\bar{p}\right.$ on $\left.\Gamma_{1} \times I\right\} \quad$ and $V_{f}=\left\{w \mid w \in H^{1}\left(\Omega_{f}\right), w=0\right.$ on $\left.\Gamma_{1}\right\}$. 
In a similar manner to that in the FEM, in the SFEM formulation, the subdomain $\Omega_{f}$ is also partitioned into nel nonoverlapping elements $\Omega_{f_{n}}$, i.e., $\Omega_{f}^{h}=\cup_{n=1}^{n e l} \Omega_{f_{n}}$ and $\Omega_{f_{n}} \cap_{\forall n \neq n^{\prime}} \Omega_{f_{n^{\prime}}}=\emptyset$. Hence, there is a diffeomorphism that preserves the orientation called mapping function, defined as $\mathcal{F}: \Lambda \rightarrow \Omega_{f_{n}}$, which relates each element $\Omega_{f_{n}}$ with the reference closed element domain $\Lambda$; in this case a biunitary square $\Lambda=[-1,1]^{2}$.

Defining $S_{f}^{h} \subset S_{f}$ and $V_{f}^{h} \subset V_{f}$ to be the finite element spaces, and $\mathbb{P}(\Lambda)$ to be the space generated by the tensor product of the Lagrange polynomials with degree $\leq m$, we have:

$$
\begin{aligned}
S_{f}^{h}= & \left\{p^{h} \mid p^{h}(\cdot, t) \in H^{1}\left(\Omega_{f}\right), p^{h}=\bar{p}^{h} \text { on } \Gamma_{1} \times I\right. \\
& \text { and } \left.\left.p^{h}\right|_{\Omega_{f_{n}}} \circ \mathcal{F} \in \mathbb{P}(\Lambda)\right\} \\
V_{f}^{h}= & \left\{w^{h} \mid w^{h} \in H^{1}\left(\Omega_{f}\right), w^{h}=0 \text { on } \Gamma_{1}\right. \\
& \text { and } \left.\left.w^{h}\right|_{\Omega_{f_{n}}} \circ \mathcal{F} \in \mathbb{P}(\Lambda)\right\}
\end{aligned}
$$

An important characteristic of the SFEM is the choice of the points for the construction of the interpolation functions. In contrast to the standard FEM with degree $m$ (i.e., equidistant nodal distributions), in the SFEM, the local nodal points are obtained by the tensor product of the $m+1$ Gauss-LobattoLegendre (GLL) points localized in the interval $[-1,1]$, defined as the roots of the equation $\frac{\partial P_{m}}{\partial \xi}\left(\xi^{2}-1\right)=0$, in which $P_{m}$ is the $m$ th Legendre polynomial and resulting in $(m+1)^{2}$ points [26,24]. Fig. 1 illustrates some spectral finite elements according to the degree $m$.

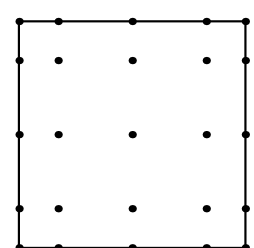

(a)

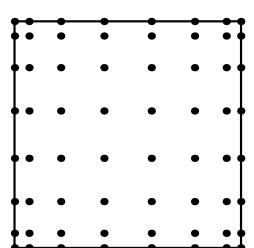

(b)

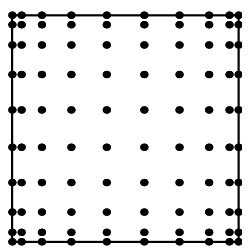

(c)

Fig. 1: 2D spectral elements with degree $m$ : (a) $4 \times 4$; (b) $7 \times 7$; (c) $9 \times 9$.

In this way, the local interpolation functions $N_{i}^{e}$ in $\Lambda$ are obtained by the tensor product of the Lagrange polynomials of degree $m$ using the $m+1 \mathrm{GLL}$ points in each direction with the relation $N_{i}^{e}\left(\boldsymbol{\xi}_{j}\right)=\delta_{i j}, i, j=1, \ldots,(m+1)^{2}$, where $\delta_{i j}$ is the Kronecker delta operator.

In the context of $2 \mathrm{D}$ wave propagation problems (acoustic or elastic), as previously commented, one major advantage of using such spectral elements lies in the fact that only 4 or 5 nodes per wavelength are required when employing polynomials with degree between $m=5$ and $m=8$. In this sense, meshes with a smaller number of elements as well as nodes are obtained when compared to meshes with the classic 4-noded quadrilateral elements, which 
require between $15-20$ points to obtain an accurate solution. Furthermore, internal nodes of the spectral elements can be condensed in order to enhance the computational efficiency of the method.

Another important issue to be considered in the SFEM formulation is the use of a quadrature rule based on the tensor product of the unidimensional GLL formulae [7], where weights $\omega_{i, m}$ and corresponding quadrature points are defined, respectively, as $\omega_{i, m}=\frac{2}{m(m+1)} \frac{1}{P_{m}\left(\xi_{i}\right)}$ and $\xi_{i}$. Such quadrature points coincide with the GLL points also adopted in the interpolation functions, leading to a degree of precision $\leq 2 m-1$. It is important to stress that with this integration scheme the stiffness matrix, originated by the bilinear form $a(\cdot, \cdot)$ is exact integrated, and all matrices originated from the $L^{2}$-inner product $(\cdot, \cdot)$ (see Eq. (8)) are naturally diagonal [26], and even that the integration doesn't be exact for the mass matrix, for example, it is important to note that the mass is conserved in high order elements.

After applying the semi-discrete SFEM formulation into Eq. (8), the following ODE system is obtained:

$$
\mathbf{M}_{f} \ddot{\mathbf{P}}+\mathbf{C}_{f} \dot{\mathbf{P}}+\mathbf{K}_{f} \mathbf{P}=\mathbf{F}_{f}+\mathbf{F}_{4}
$$

where $\mathbf{M}_{f} \in \mathbb{R}^{n q_{f} \times n q_{f}}, \mathbf{C}_{f} \in \mathbb{R}^{n q_{f} \times n q_{f}}$ and $\mathbf{K}_{f} \in \mathbb{R}^{n q_{f} \times n q_{f}}$ denote the standard mass, damping (due to the absorbing boundary condition) and stiffness matrices, respectively, the vectors $\mathbf{P}: I \rightarrow \mathbb{R}^{n q_{f}}, \dot{\mathbf{P}}: I \rightarrow \mathbb{R}^{n q_{f}}, \ddot{\mathbf{P}}: I \rightarrow \mathbb{R}^{n q_{f}}$, $\mathbf{F}_{f}: I \rightarrow \mathbb{R}^{n q_{f}}$ and $\mathbf{F}_{4}: I \rightarrow \mathbb{R}^{n q_{f}}$ represent, respectively, the nodal hydrodynamic pressures, their first and second time derivatives, external forces and coupling forces acting on the fluid subdomain due to the interaction with the solid subdomain, with $n q_{f}$ being the number of equations in the fluid model. The numerical treatment of $\mathbf{F}_{4}$ is also presented in the section 3.

\subsection{The HHT time-marching scheme}

The obtained ODE systems given by Eqs. (4) and (10) can be recast in a general form as follows:

$$
\mathbf{M Z}+\mathbf{C Z}+\mathbf{K Z}=\mathbf{F}
$$

where the vector $\mathbf{Z}$ corresponds either to $\mathbf{U}$ or $\mathbf{P}$, according to the subdomain in focus, as well as the respective matrices, e.g., for the solid subdomain the matrix $\mathbf{C}=\mathbf{0}$, once no absorbing boundary conditions and/or viscous damping are prescribed.

In this work, the ODE systems plus proper initial conditions are solved by the HHT time-marching scheme with different parameters for each the solid subdomain and the fluid one whose reasons are explained later on. When standard time-marching schemes are employed, the analysis time $I$ needs to be firstly partitioned into $L_{z}$ time subintervals (assumed constant here), i.e., $[0, T]=\cup_{l=0}^{L_{z}-1}\left[t_{z, l}, t_{z, l+1}\right]$, with $0=t_{z, 0}<\ldots<t_{z, L_{z}}=T, \Delta t_{z}=t_{z, l+1}-$ $t_{z, l}=T / L_{z}$ and $t_{z, l+1}=(l+1) \Delta t_{z}$ so that $\mathbf{Z}_{n}^{l+1}=\mathbf{Z}_{n}\left(t_{l+1}\right)$, where the index $z$ is related to the fluid or solid subdomain. 
In the HHT scheme, Eq. (11) turns as follows:

$$
\begin{aligned}
\overline{\mathbf{M}} \ddot{\mathbf{Z}}^{l+1} & =\mathbf{F}^{l+\alpha}-(1+\alpha)\left(\mathbf{C} \tilde{\mathbf{Z}}^{l+1}+\mathbf{K} \tilde{\mathbf{Z}}^{l+1}\right)+\alpha\left(\mathbf{C} \dot{\mathbf{Z}}^{l}+\mathbf{K} \mathbf{Z}^{l}\right) \\
\overline{\mathbf{M}} & =\left(\mathbf{M}+(1+\alpha)\left(\gamma \Delta t_{z} \mathbf{C}+\beta \Delta t_{z}^{2} \mathbf{K}\right)\right)
\end{aligned}
$$

where $\mathbf{F}^{l+\alpha}=\mathbf{F}\left(t_{z, l+\alpha}\right), t_{z, l+1+\alpha}=(1+\alpha) t_{z, l+1}-\alpha t_{z, l}=t_{z, l+1}+\alpha \Delta t_{z}$ and the predictors vectors are defined as:

$$
\begin{aligned}
& \tilde{\mathbf{Z}}^{l+1}=\mathbf{Z}^{l}+\Delta t_{z} \dot{\mathbf{Z}}^{l}+\frac{\Delta t_{z}^{2}}{2}(1-2 \beta) \ddot{\mathbf{Z}}^{l} \\
& \tilde{\dot{\mathbf{Z}}}^{l+1}=\dot{\mathbf{Z}}^{l}+(1-\gamma) \Delta t_{z} \ddot{\mathbf{Z}}^{l}
\end{aligned}
$$

while the correctors vectors are defined as:

$$
\begin{aligned}
& \mathbf{Z}^{l+1}=\tilde{\mathbf{Z}}^{l+1}+\beta \Delta t_{z}^{2} \ddot{\mathbf{Z}}^{l+1} \\
& \dot{\mathbf{Z}}^{l+1}=\tilde{\mathbf{Z}}^{l+1}+\gamma \Delta t_{z} \ddot{\mathbf{Z}}^{l+1}
\end{aligned}
$$

The HHT method can be seen as a generalization of the Newmark method, since selecting $\alpha=0$ lies in the quoted scheme [19]. In the Newmark method, the parameters $\beta$ and $\gamma$ determine the stability and accuracy characteristics, e.g., the trapezoidal rule is obtained using $\beta=\frac{1}{4}$ and $\gamma=\frac{1}{2}$, and the central difference method by using $\beta=0$ and $\gamma=\frac{1}{2}$, both of them with second order accuracy and being respectively implicit and explicit, thus possessing unconditional and conditional stability. However, numerical damping cannot be introduced in the Newmark schemes without degrading the order of accuracy. Hence, when the parameters are selected as $\left.\alpha \in\left[-\frac{1}{3}, 0\right]\right], \beta=(1-\alpha)^{2} / 4$ and $\gamma=(1-2 \alpha) / 2$, the HHT method becomes unconditionally stable and second-order accurate with a numerical damping controlled by the parameter $\alpha[18]$.

In this way, one can select the time-marching scheme according to the subdomain in focus, e.g., one can improve the accuracy of the results in the fluid subdomain using the HHT scheme with numerical damping as seen later on.

\section{Iterative multi-time step coupling method}

Generally speaking, an iterative coupling technique applied at the coupling interface consists in transfer some information of the solution from one subdomain to the other through the coupling interface, until the desired convergence of the relevant variables is attained. Hence, one can use distinct spatial discretization techniques, time-marching schemes and time step sizes for each subdomain, as well as nonconforming meshes at the coupling interface, since the coupling conditions are hold at the end of the iterative process.

This section presents the proposed FEM-SFEM iterative procedure through two subsections in order to clearly explain the benefits and distinct features 
of the proposed iterative formulation. In the first part, the methodology is restricted for the case of equal time step sizes in all the subdomains, whereas, in the second part, the use of different time step sizes, i.e., the time subcycling scheme and the differences between the traditional iterative coupling methodologies and the proposed scheme are presented.

\subsection{Spatial coupling - equal time step sizes}

When the fluid subdomain time step size is equal to the solid subdomain one (i.e. $\Delta t_{f}=\Delta t_{s}$ ), it is not necessary to distinguish the master and slave subdomains. However, hereafter the fluid subdomain is named as the master and the solid as the slave in the sense that the former is always analyzed first and it is discretized by the SFEM with large elements. In the iterative coupled procedure, the computations corresponding to the $k t h$ iterative step at the $l t h$ time step proceed as follows:

I - Computation of $\mathbf{P}^{l+1, k+1}$ - Fluid subdomain:

i - The surface fluxes on the coupling interface are computed, taking into account the discrete counterparts of the last terms of Eqs. (7)-(8), with the already evaluated acceleration nodal values on the coupling interface of the last iterative step, i.e. $\ddot{\overline{\mathbf{U}}}^{l+1, k}$ (the overbar refers to coupling interface), leading to:

$$
\mathbf{F}_{4}^{l+1, k+1}=\int_{\Gamma_{4}} \mathbf{N}_{f}^{T} \kappa_{f} q^{l+1, k} d \Gamma=-\kappa_{f} \rho_{f} \int_{\Gamma_{4}} \mathbf{N}_{f}^{T} \mathbf{n}_{f}^{T} \mathbf{N}_{s} \ddot{\overline{\mathbf{U}}}^{l+1, k} d \Gamma
$$

From now on $\mathbf{N}_{z}$ stands for the fluid (SFEM) or solid (FEM) interpolation functions, according to the subscript $f$ or $s$, respectively, and $\mathbf{n}_{f}$ represents the fluid subdomain outward normal vector. The above boundary integral is calculated numerically by a mapping and interpolation procedures as described in detail next.

ii - The new pressure nodal values $\mathbf{P}^{l+1, k+1}$ concerning the fluid subdomain are computed in a coupled form by Eqs. (10)-(12).

II - Computation of $\ddot{\mathbf{U}}^{l+1, k+1}$ - Solid subdomain:

$\mathrm{i}$ - The interaction forces on the coupling interface are computed, taking into account the discrete counterparts of the last terms of Eqs. (2)-(3), with the pressure nodal values on the coupling interface obtained from step I, i.e. $\overline{\mathbf{P}}^{l+1, k+1}$, yielding:

$$
\mathbf{F}_{T}^{l+1, k+1}=\int_{\Gamma_{T}} \mathbf{N}_{s}^{T} \boldsymbol{\tau}^{l+1, k+1} d \Gamma=\int_{\Gamma_{T}} \mathbf{N}_{s}^{T} \mathbf{n}_{f} \mathbf{N}_{f} \overline{\mathbf{P}}^{l+1, k+1} d \Gamma
$$

ii - The new acceleration nodal values, denoted by $\ddot{\mathbf{U}}^{l+1, k+1}$, concerning the solid subdomain are computed in a coupled form by Eqs. (4) and (12). 
iii - Traditionally, in this step a relaxation scheme is performed at the coupling interface for the already evaluated accelerations in order to ensure and/or to speed-up the convergence of the iterative process, i.e.:

$$
\ddot{\overline{\mathbf{U}}}^{l+1, k+1}=\lambda \ddot{\overline{\mathbf{U}}}^{l+1, k+\lambda}+(1-\lambda) \ddot{\overline{\mathbf{U}}}^{l+1, k}
$$

where $\ddot{\overline{\mathbf{U}}}^{l+1, k+\lambda}$ stands for the already evaluated accelerations (nonrelaxed) at the coupling interface.

iv - Finally, the relaxation parameter $\lambda$ is updated in an adaptive manner to be used in the next iteration, as described later on.

Once the convergence criterion is attained inside the time step, the nodal vectors $\mathbf{U}, \dot{\mathbf{U}}, \dot{\mathbf{P}}$ are updated in their respective subdomains, and the above procedure is reinitialized for the next time step.

It is worth pointing out that in the initialization of the iterative procedure (first iterative step), the fluid subdomain is analyzed in an isolated manner in which surface fluxes on the coupling interface are considered null (see Eq. (15) with null accelerations), such that the solid subdomain can be then analyzed in a coupled form according to Eq. (16). The adopted convergence criterion for the iterative process is defined as $\frac{\left\|\ddot{\overline{\mathbf{U}}}^{l+1, k+1}-\ddot{\overline{\mathbf{U}}}^{l+1, k}\right\|}{\left\|\ddot{\overline{\mathbf{U}}}^{l+1, k+1}\right\|}<\epsilon$, with $\epsilon$ being a given tolerance.

\subsubsection{Integrals on the coupling surface}

In order to correctly evaluate the integrals in Eqs. (15)-(16), an inverse mapping followed by an interpolation procedure is employed. Focusing on the boundary integral (16) for instance, the following steps are applied: i) Gauss integration points are mapped from the reference domain to the physical element boundary $\Gamma_{T n}$; ii) once the coordinates of the integration points in the physical boundary are known, the corresponding element boundary $\Gamma_{4 n}$ of a spectral element $\Omega_{f n}$ is readily identified; iii) once the spectral element is known, an inverse mapping procedure with respect to the spectral element enables us to compute the interpolated pressure field along $\Gamma_{4 n}$ adopting the one-dimensional SFEM basis functions in the reference domain. This scheme is illustrated in Figure 2 for both integrals (notice that for the SFEM the GLL integration points coincide with the nodal points).

Note that the inverse mapping function for one side of an isoparametric bilinear quadrilateral element (FEM) falls into the 1D case and can be defined as $\mathcal{F}^{-1}: \Gamma_{T n} \rightarrow \Lambda_{1 D}$, where $\Gamma_{T n}$ stands for the side of the element $\Omega_{s n}$ at $\Gamma_{T n}$ and $\Lambda_{1 D}=[-1,1]$. Thus, let $\mathbf{x}_{e}=(x, y)$ be a mapped integration point from $\Gamma_{4 n} \rightarrow \Gamma_{T n}$ (see Fig. 2a), the corresponding inverse mapping from $\Gamma_{T n} \rightarrow \Lambda_{1 D}$ is given by $\xi=\frac{2(x+y)-\left(x_{1}+x_{2}+y_{1}+y_{2}\right)}{x_{2}-x_{1}+y_{2}-y_{1}}$, where $\mathbf{x}_{e_{1}}=\left(x_{1}, y_{1}\right)$ and $\mathbf{x}_{e_{2}}=\left(x_{2}, y_{2}\right)$ are the nodal coordinates of $\Omega_{s_{n}}$ at $\Gamma_{T}$.

Conversely, using a subparametric approximation for the SFEM (i.e., interpolation functions of high degree but bilinear quadrilateral elements), the 


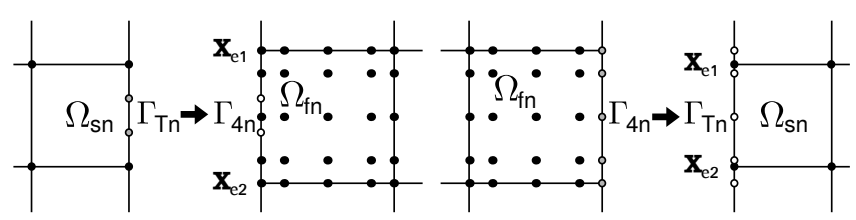

(a) From $\Omega_{s}$ to $\Omega_{f}$. (b) From $\Omega_{f}$ to $\Omega_{s}$.

Fig. 2: Sketch of the inverse mapping of the integration points; the inverse mapped points are represented by the void points, the black points represent the mesh nodes and the gray points represent the integration points.

inverse mapping falls into the same case previously discussed. Although this subparametric approximation is adopted here, if a better representation of the geometry boundary is required, more complex mapping schemes could also be employed (e.g., blending type functions) [38]. In this sense, the aforementioned discussed mapping scheme for the integration points remains almost the same, and the only difference lies in the fact that the inverse mapping must be carried out by means of the Newton-Raphson method, for instance.

\subsubsection{Optimal relaxation parameter}

In this work, a relaxation parameter based on the minimization of the square error functional of the relevant variables at the coupling interface described in $[33,34,36,15]$ is also implemented in order to compare with the proposed methodology. In this way, the square error functional concerning the solid accelerations in the time step $l+1$ between two successive iterative steps $k+1$ and $k$ is considered, namely:

$$
\mathcal{L}(\lambda)=\left\|\ddot{\mathbf{U}}^{l+1, k+1}-\ddot{\overline{\mathbf{U}}}^{l+1, k}\right\|^{2}
$$

where $\ddot{\overline{\mathbf{U}}}^{l+1, k+1}=\lambda \ddot{\overline{\mathbf{U}}}^{l+1, k+\lambda}+(1-\lambda) \ddot{\overline{\mathbf{U}}}^{l+1, k}$ and $\ddot{\overline{\mathbf{U}}}^{l+1, k}=\lambda \ddot{\overline{\mathbf{U}}}^{l+1, k+\lambda-1}+$ $(1-\lambda) \ddot{\overline{\mathbf{U}}}^{l+1, k-1}$ are the relaxed accelerations and $\ddot{\overline{\mathbf{U}}}^{l+1, k+\lambda}$ and $\ddot{\overline{\mathbf{U}}}^{l+1, k+\lambda-1}$ the non-relaxed accelerations (previously introduced), giving after some math:

$$
\lambda=\frac{\left(\mathbf{W}^{k}, \mathbf{W}^{k}-\mathbf{W}^{k+\lambda}\right)}{\left\|\mathbf{W}^{k}-\mathbf{W}^{k+\lambda}\right\|^{2}}
$$

where $(\cdot, \cdot)=\|\cdot\|^{2}$ and with the variables $\mathbf{W}^{k+\lambda}=\ddot{\overline{\mathbf{U}}}^{l+1, k+\lambda}-\ddot{\overline{\mathbf{U}}}^{l+1, k+\lambda-1}$ and $\mathbf{W}^{k}=\ddot{\overline{\mathbf{U}}}^{l+1, k}-\ddot{\overline{\mathbf{U}}}^{l+1, k-1}$.

Notice that the obtained expression possesses a simple implementation and a low computational cost $[36,15]$; besides, a value close to one, say $\lambda=0.9$, is employed at the first iteration step. 
3.2 Time subcycling - different time steps

In this work, a different way to improve the efficiency of the algorithm avoiding the computation of a relaxation parameter is presented; it is important to note that such a scheme is applicable only in the case of different time-step sizes for the fluid and solid subdomains, i.e, the original scheme with relaxation is used when equal time step sizes are used. In this sense, with the possibility of using different time-step sizes in each subdomain, one can adopt an ideal time-step size for each subdomain, improving the overall efficiency of the algorithm. In a great deal of cases for solid-fluid coupling problems, the solid P-wave velocity can be larger than that of the fluid, requiring smaller time steps within the solid subdomain. Thus, considering $\Delta t_{s} \leq \Delta t_{f}$, and assuming that $t_{f, l}<t_{s, l+1}<t_{f, l+1}$, the steps presented below must be appended to those described in section 3.1 to evaluate the boundary integrals:

- $\ddot{\overline{\mathbf{U}}}^{\left.(l+1)\right|_{s}, k+1}$ extrapolation - Fluid subdomain:

i- In order to correctly evaluate the boundary integral (15) on the fluid subdomain, a constant time extrapolation for the acceleration nodal values is performed. In this work, it is proposed that only the first iterative step values are extrapolated replacing $\ddot{\overline{\mathbf{U}}}^{l+1, k}$ by $\ddot{\overline{\mathbf{U}}}^{l+\left.1\right|_{f}, 1}$ in Eq. (15) and yielding:

$$
\ddot{\overline{\mathbf{U}}}^{\left.(l+1)\right|_{f}, k+1}=\ddot{\overline{\mathbf{U}}}^{\left.(l+1)\right|_{s}, 1}
$$

Thus instead of transferring to the fluid subdomain the acceleration nodal values at the current iterative step, just the first computed nodal values at the coupling interface are actually transferred (a sketch of the extrapolation scheme can be seen in Figure 3(a)). Note that the amount of times that a new acceleration information $\ddot{\overline{\mathbf{U}}}^{l+\left.1\right|_{f}, 1}$ is transferred to the fluid subdomain depends on the amount of subcycling steps adopted, i.e. if $\Delta t_{f}=a \Delta t_{s}$, a new acceleration information is transferred $a$ times until the time-step sizes of both subdomains coincide or when $t_{s, l+1}=t_{f, l+1}$.

ii - The time marching in the fluid subdomain only advances in time when the solid time instant coincides with the fluid one (i.e. $t_{s, l+1}=t_{f, l+1}$ ). Then, the pressure vector $\dot{\mathbf{P}}$ is updated and the iterative process is repeated for the next time step.

- $\overline{\mathbf{P}}^{\left.(l+1)\right|_{s}, k+1}$ interpolation - Solid subdomain:

i - In order to correctly evaluate the boundary integral (16) in the solid subdomain, the pressure nodal values, already obtained, are linearly time interpolated between the values of the previous time step $\overline{\mathbf{P}}^{\left.l\right|_{f}}$ and those of the current iterative step at the current time step, i.e.:

$$
\overline{\mathbf{P}}^{\left.(l+1)\right|_{s}, k+1}=(1-\varepsilon) \overline{\mathbf{P}}^{\left.l\right|_{f}}+\varepsilon \overline{\mathbf{P}}^{\left.(l+1)\right|_{f}, k+1}
$$


where $\varepsilon=\frac{t_{s, l+1}-t_{f, l}}{\Delta t_{f}}$. In Figure 3(b) one can see a sketch of the interpolation scheme. In this way, the boundary integral (16) is computed replacing $\overline{\mathbf{P}}^{(l+1), k+1}$ by $\overline{\mathbf{P}}^{\left.(l+1)\right|_{s}, k+1}$ of the above expression.

ii - Once convergence is attained, the other nodal value vectors, i.e. $\mathbf{U}$ and $\dot{\mathbf{U}}$, are updated and the subcycling step for the solid subdomain is initialized.

By using the proposed simple extrapolation scheme given by Eq. (20), a number of advantages can be highlighted, namely: i) the steps II-iii and II-iv described in section 3.1 are skipped, i.e., the adoption of a relaxation parameter is no more necessary, ii) very straightforward and efficient computer implementation, and iii) the convergence in the proposed methodology is attained through a smaller number of iterative steps as described in the numerical examples.

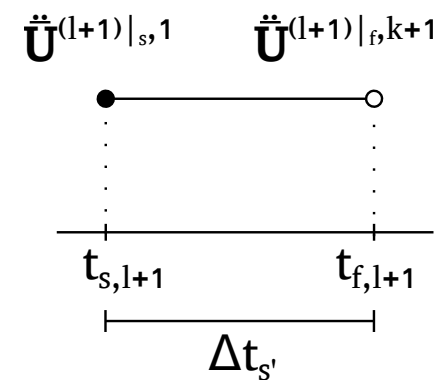

(a) $\left.\ddot{\mathbf{U}}^{(l+1)}\right|_{s}, 1$ extrapolation.

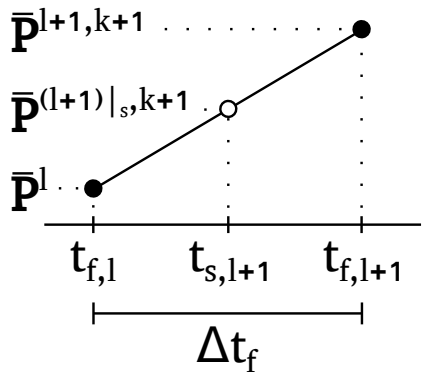

(b) $\overline{\mathbf{P}}^{\left.(l+1)\right|_{s}, k+1}$ interpolation.

Fig. 3: Sketch of the time interpolation/extrapolation scheme with the black points being the known values and the void point being the interpolated value, note that in the subfigure (a) $\Delta t_{s^{\prime}}=t_{f, l+1}-t_{s, l+1}$.

\section{Numerical simulations}

Two numerical examples are presented in order to illustrate the effectiveness and potentialities of the proposed iterative methodology. The first example consists in a solid-fluid column under a load at one of its extremes and clamped at the other one, in which the subcycling scheme is analyzed varying the time step sizes on the subdomains. Then, for the second example, a more complex problem, represented by a dam retaining water of a storage-lake is studied. For all the applications that follow, the trapezoidal rule (i.e., $\alpha=0, \beta=0.25$ and $\gamma=0.50)$ is considered for the solid subdomain, whereas the HHT scheme with numerical damping by selecting $\alpha=-\frac{1}{3}$ is adopted for the fluid subdomain. In all cases, the FEM-FEM standard method with matching nodes at the coupling interface (conforming mesh) with the trapezoidal rule is considered 
as the reference solution. Moreover, the tolerance used in the iterative coupling procedure is set to $\epsilon=10^{-7}$.

\subsection{Steel-water column}

This numerical example consists in a steel-water column with dimensions $a=$ $4.0 \mathrm{~m}$ and $b=1.0 \mathrm{~m}$ subjected to a Heaviside load acting at one of its ends, as depicted in Figure 4. As one can see, the water subdomain is discretized by 4 spectral elements with degree $m=7$, resulting in a total of 225 nodes, whereas the steel subdomain is discretized by 200 four-noded quadrilateral elements, resulting in 231 nodes.

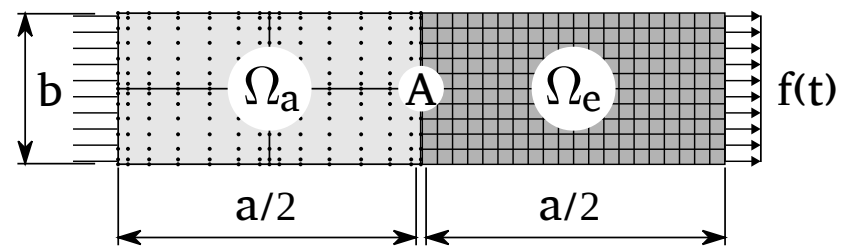

Fig. 4: Sketch and discretization of the steel-water column.

The material properties of the media are: $\kappa_{f}=2.3175 \times 10^{9} \mathrm{~N} / \mathrm{m}^{2}$ (bulk modulus); $\rho_{f}=1030 \mathrm{~kg} / \mathrm{m}^{3}$ (density); $E=2.1 \times 10^{11} \mathrm{~N} / \mathrm{m}^{2}$ (Young modulus); $\nu=0.3$ (Poisson ratio); $\rho_{s}=7700 \mathrm{~kg} / \mathrm{m}^{3}$, resulting in a model with different wave propagation velocities within the solid and fluid subdomains, i.e., $v_{P}=$ $6059 \mathrm{~m} / \mathrm{s}$ (P-wave) and $v_{S}=3239 \mathrm{~m} / \mathrm{s}$ (S-wave) for the solid and $v_{f}=1500 \mathrm{~m} / \mathrm{s}$ for the fluid.

In Figure 5, the displacement and hydrodynamic pressure time-history results at point $A=(a / 2, b / 2)$ (see Fig. 4) are depicted, considering three cases; namely, $\Delta t_{f}=\Delta t_{s}, \Delta t_{f}=3 \Delta t_{s}$ and $\Delta t_{f}=6 \Delta t_{s}$ where $\Delta t_{s}=3.0 \times 10^{-6} \mathrm{~s}$, as well as the reference solution. Recall that the procedure for the case when $\Delta t_{f}=\Delta t_{s}$ has been presented in section 3.1; besides, the adaptive relaxation parameter has been implemented for this case. For the remaining cases the proposed scheme has been employed.

Note that spurious oscillations appear on the reference solution of Figure 5(b) due to the fact that the trapezoidal rule is used in both subdomains, which can lead to the misinterpretation of the results. Thus, the importance of the addition of the HHT method is clearly observed, since the order of accuracy is maintained as the same time that a controlled numerical damping is introduced.

As one can see, the number of subcycling steps apparently does not influence considerably the accuracy of the results, since no loss of precision is observed even when $\Delta t_{f}=6 \Delta t_{s}$, while the computational efficiency was improved. It is worth pointing out that the results presented here are in good agreement with those found in the literature $[37,34]$. 


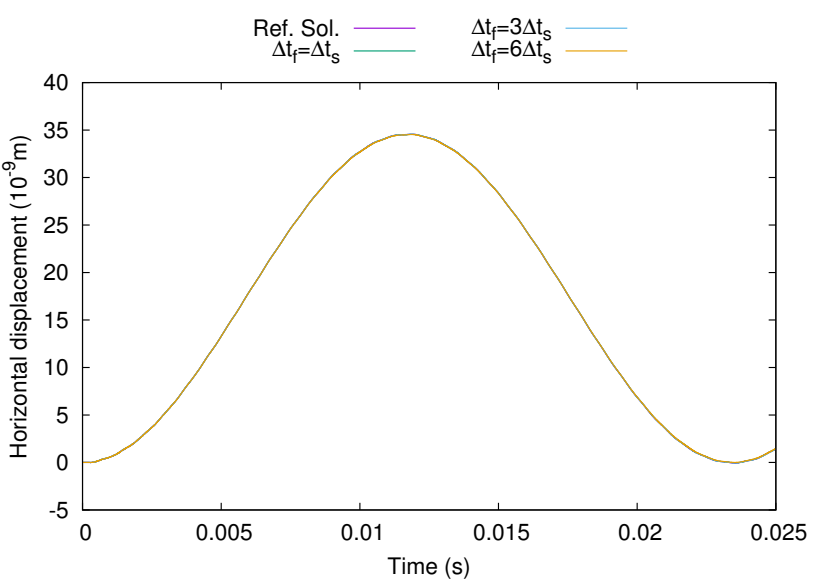

(a) Horizontal displacements.

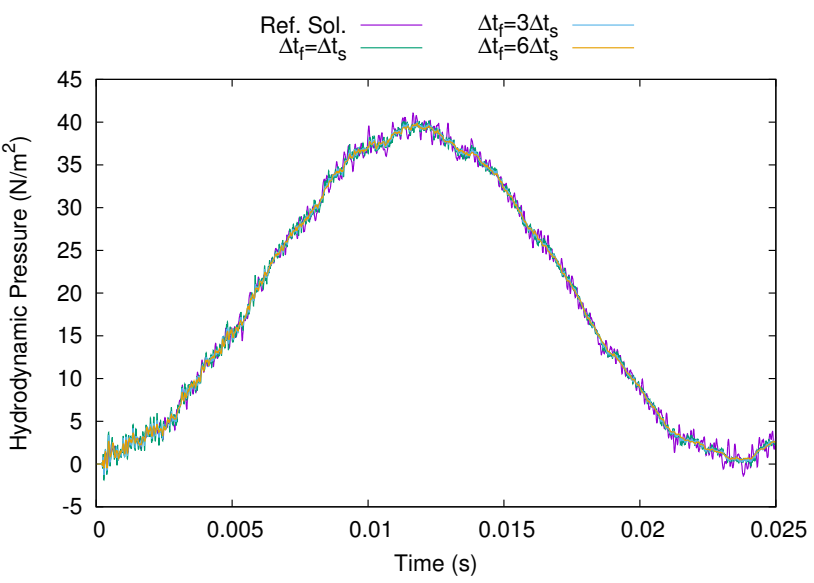

(b) Hydrodynamic pressures.

Fig. 5: Time-history results for the steel-water column at point $A$ considering distinct time steps for each subdomain.

\subsection{Dam-reservoir system}

The dam-reservoir system depicted in Figure 6 is now analyzed. The dam is subjected to a uniformly distributed dynamic load of sinusoidal type at the top, acting with an angular frequency of $\omega=18 \mathrm{rad} / \mathrm{s}$ (i.e. $f(t)=\sin (\omega t)$ ). The material properties of the dam are: $E=3.437 \times 10^{9} \mathrm{~N} / \mathrm{m}^{2}, \nu=0.25$ and $\rho_{s}=2000 \mathrm{~kg} / \mathrm{m}^{3}$. The adjacent water is characterized by $v_{f}=1436 \mathrm{~m} / \mathrm{s}$ and $\rho=1000 \mathrm{~kg} / \mathrm{m}^{3}$. In order to model the contact of water with air, the hydrodynamic pressure is set to zero along the top, whereas at the bottom of the reservoir (wall surface), a rigid boundary condition (i.e. zero flux) is 
prescribed. Moreover, along with the right side, the viscous damping absorbing boundary condition [28] as seen in Eq. (7) is applied to model the unbounded domain. The time-step sizes adopted for the analysis are $\Delta t_{f}=\Delta t_{s}, \Delta t_{f}=$ $3 \Delta t_{s}$ and $\Delta t_{f}=6 \Delta t_{s}$ where $\Delta t_{s}=3.5 \times 10^{-4} \mathrm{~s}$.

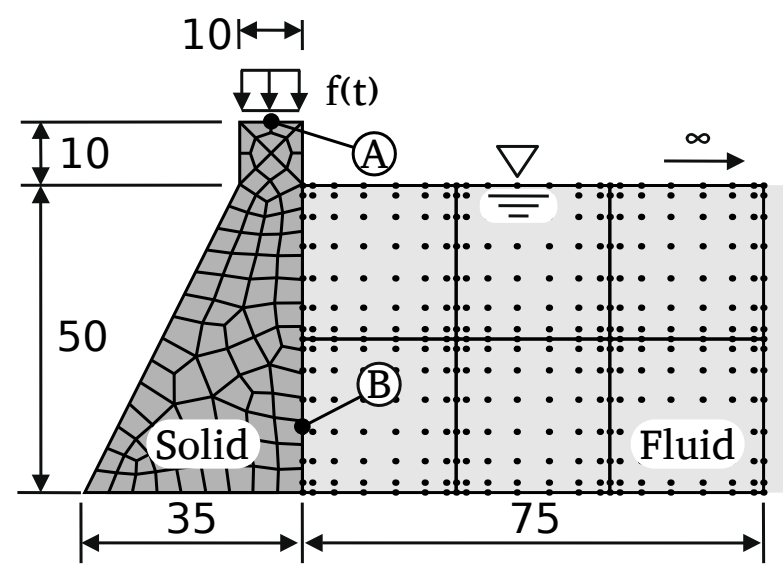

Fig. 6: Geometry and loading of the dam-reservoir system, adapted from [35].

The mesh adopted for the acoustic fluid consists in 6 spectral elements with degree $m=7$, resulting in 330 nodes, while for the solid a mesh with 81 quadrilateral bilinear elements and 103 nodes is employed. The use of the SFEM in the acoustic subdomain lies in the fact that only 4-5 nodes per wavelength are required to generate accurate results in the acoustic subdomain in contrast to the 15-20 nodes required if the standard FEM was employed.

Figure 7 depicts the vertical displacement and the hydrodynamic pressure time-history results at points $A=(30,60)$ and $B=(35,10)$. As one can see, accurate results are obtained by the present FEM-SFEM iterative coupling procedure when compared to the reference solution [14]. The results are also in accordance with those from the literature [35,37].

In Figure 8, snapshots of the horizontal displacement and the pressure at different time instants are plotted for the corresponding subdomains to show the overall behavior of the coupled system due to the interaction as time advances. In Figures 8(a)-(b), the presence of a pressure wavefront at the fluid subdomain originated from the dynamic interaction with the dam is clearly visible. Only the horizontal displacement is shown here since the influence of the fluid on the dam occurs in the horizontal direction through the pressure on the coupling interface.

In order to better analyze the overall performance of the proposed formulation, Figure 9 depicts a percentage analysis of the number of iterations required for convergence in each time step for the whole analysis consisted of 2000 time steps (recall that $\Delta t_{s}=3.5 \times 10^{-4} \mathrm{~s}$ with the analysis time terminating at $0.7 \mathrm{~s}$ ). The graphics are related to three different methodologies; namely, the itera- 


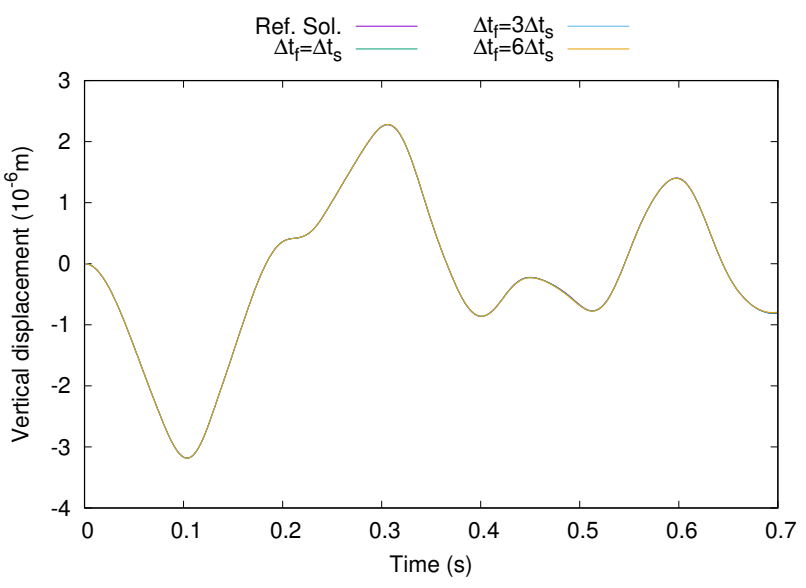

(a) Vertical displacements at point $A$.

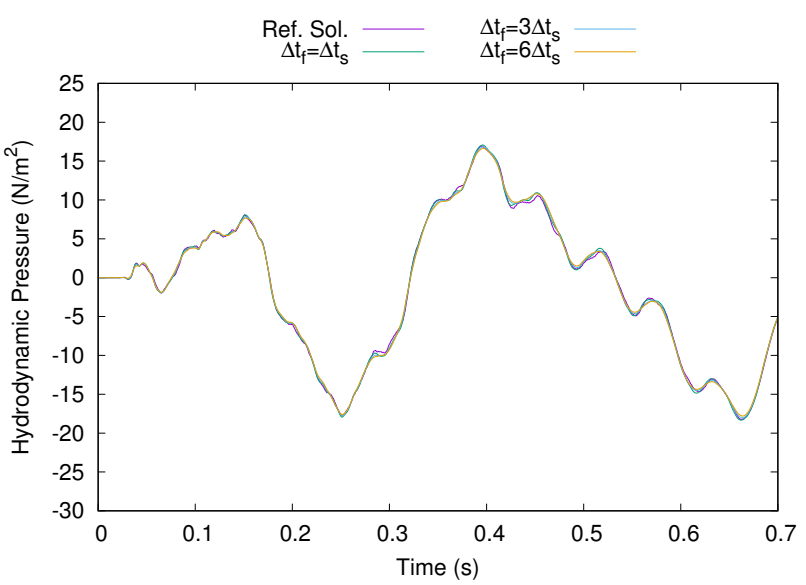

(b) Hydrodynamic pressures at point $B$.

Fig. 7: Time-history results for the dam reservoir.

tive coupling without relaxation in blue, the one with an adaptive relaxation parameter in orange (see subsection 3.1.2), and the proposed scheme in yellow. In Fig.9(a) one can see the percentage distribution for the case of $\Delta t_{f}=2 \Delta t_{s}$, in which an amount of 8 iterations is required to attain convergence for more than $60 \%$ of the time steps with the proposed scheme (in yellow). On the other hand, considering the adaptive relaxation parameter, 10 iterations are required for more than $45 \%$ of the time steps. Conversely, it is readily observed that the number of iterations clearly increases when a relaxation strategy is not considered; besides, the number of iterations is more spread around the value 24 , indicating a lack of consistence for attaining convergence. 


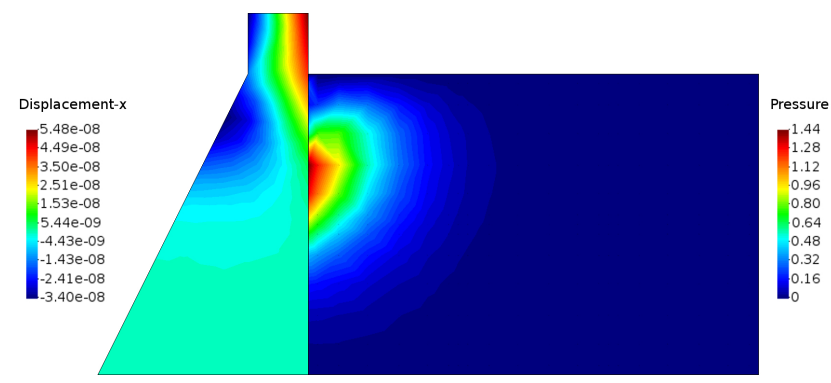

(a) Snapshot at t $=0.028 \mathrm{~s}$.

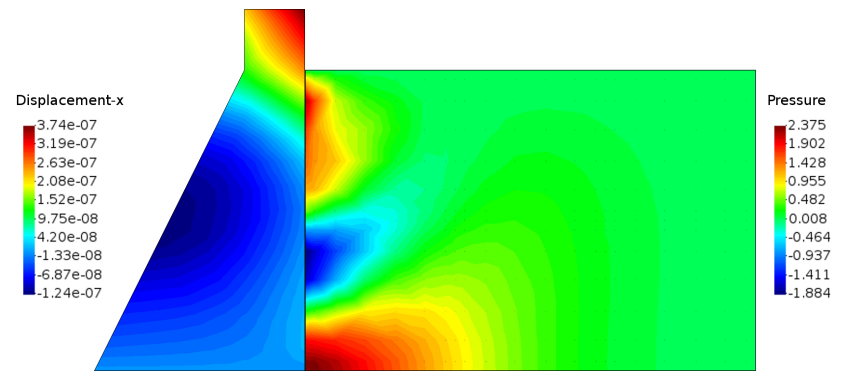

(b) Snapshot at $\mathrm{t}=0.056 \mathrm{~s}$

Fig. 8: Snapshots of the horizontal displacement and pressure

Analyzing Fig.9(b) for the case of $\Delta t_{f}=6 \Delta t_{s}$, one can see that the total number of iterations required to converge is directly proportional to the difference between the time-step sizes of the subdomains as expected. In fact, compared to the previous case, an amount of 14 iterations is now required for the proposed scheme to attain convergence for more than $40 \%$ of the time steps with the remaining ones being concentrated in the range of [11-15] iterations. Moreover, the proposed scheme performs much better with overall less iterations than the scheme with adaptive relaxation parameter in the sense that for the latter the number of iterations varies greatly from 12 to 24 iterations. Differently from the previous case, now without the use of the relaxation parameter, the solution does not converge at all.

\section{Conclusions}

In this work, an efficient multi-time step FEM-SFEM iterative coupling procedure for the time-domain analysis of coupled solid-fluid problems has been presented. By means of the iterative coupling procedure, the FEM-SFEM methodology reveals a great potential since the use of nonconforming meshes at the coupling interface allows a more flexibility in the mesh generation especially for the SFEM. In this way, regular spectral elements can be more easily generated, allowing to make full use of the high accuracy of the SFEM. Due to the 


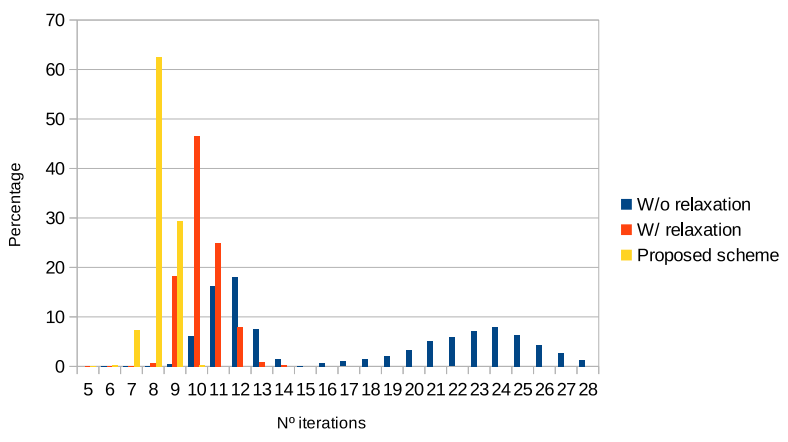

(a) $\Delta t_{f}=2 \Delta t_{s}$

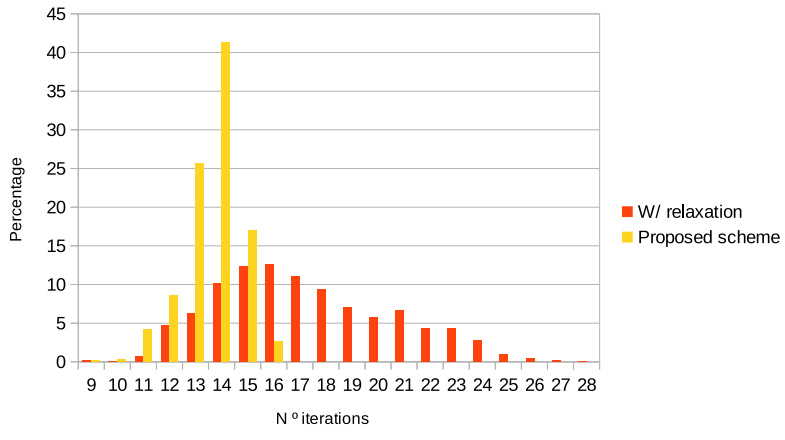

(b) $\Delta t_{f}=6 \Delta t_{s}$

Fig. 9: Distribution of the number of iterations at each time step for the whole analysis.

independent treatment of the subdomains, one can adopt adequate time-step sizes for each subdomain (subcycling), as well as time integration schemes and solvers, resulting in an even more efficient and accurate methodology. Furthermore, by a modification in the subcycling procedure, the efficiency of the methodology is improved, avoiding the computation of a relaxation parameter when different time-steps sizes are used. The numerical results presented here illustrate the good accuracy of the proposed methodology. Finally, regarding all advantages discussed here for the proposed methodology, it is expected that straightforward computer implementation and accurate results may be also attained for more complex problems involving other solid-fluid models, as well as other types of similar coupled problems.

Acknowledgements The financial support by CAPES, UFJF, UFSJ, FAPEMIG and $\mathrm{CNPq}$ is greatly acknowledged. 


\section{References}

1. Adams, R., Fournier, J.: Sobolev Spaces, 2 edn. Pure and Applied Mathematics. Elsevier Science (2003)

2. Bathe, K.: Finite element procedures. Prentice-Hall, New Jersey (1996)

3. Belytschko, T., Mullen, R.: Stability of explicit-implicit mesh partitions in time integration. International Journal for Numerical Methods in Engineering 12(10), 1575-1586 (1978)

4. Belytschko, T., Yen, H.J., Mullen, R.: Mixed methods for time integration. Computer Methods in Applied Mechanics and Engineering 17-18, Part 2, 259-275 (1979)

5. Bernardi, C., Maday, Y., Patera, A.T.: Domain Decomposition by the Mortar Element Method, pp. 269-286. Springer Netherlands (1993)

6. Boyd, J.: Chebyshev and Fourier Spectral Methods: Second Revised Edition. Dover Publications (2001)

7. Canuto, C., Hussaini, Y., Quarteroni, A., Zang, T.A.: Spectral Methods: Fundamentals in Single Domains. Springer (2006)

8. Canuto, C., Hussaini, Y., Quarteroni, A., Zang, T.A.: Spectral Methods: Evolution to Complex Geometries and Applications to Fluid Dynamics. Springer (2007)

9. Casadei, F., Gabellini, E., Fotia, G., Maggio, F., Quarteroni, A.: A mortar spectral/finite element method for complex 2D and 3D elastodynamic problems. Computer Methods in Applied Mechanics and Engineering 191(45), 5119-5148 (2002)

10. Coulier, P., François, S., Lombaert, G., Degrande, G.: Coupled finite element - hierarchical boundary element methods for dynamic soil-structure interaction in the frequency domain. International Journal for Numerical Methods in Engineering 97(7), 505-530 (2014)

11. El-Gebeily, M., Elleithy, W.M., Al-Gahtani, H.J.: Convergence of the domain decomposition finite elementboundary element coupling methods. Computer Methods in Applied Mechanics and Engineering 191(43), 4851 - 4867 (2002)

12. Elleithy, W., Al-Gahtani, H., El-Gebeily, M.: Iterative coupling of BE and FE methods in elastostatics. Engineering Analysis with Boundary Elements 25(8), 685-695 (2001)

13. Elleithy, W.M., Tanaka, M.: Interface relaxation algorithms for bembem coupling and fembem coupling. Computer Methods in Applied Mechanics and Engineering 192(26), $2977-2992(2003)$

14. von Estorff O., Antes, H.: On FEM-BEM coupling for fluid-structure interaction analyses in the time domain. International Journal for Numerical Methods in Engineering 31(6), 1151-1168 (1991)

15. Fontes Jr, E., Santiago, J., Telles, J.: An iterative coupling between meshless methods to solve embedded crack problems. Engineering Analysis with Boundary Elements 55 $52-57(2015)$

16. François, S., Coulier, P., Degrande, G.: Finite element-boundary element coupling algorithms for transient elastodynamics. Engineering Analysis with Boundary Elements 55, 104-121 (2015)

17. Gopalakrishnan, S., Chakraborty, A., Mahapatra, D.R.: Spectral Finite Element Method: Wave Propagation, Diagnostics and Control in Anisotropic and Inhomogeneous Structures. Springer (2007)

18. Hilber, H.M., Hughes, T.J.R., Taylor, R.L.: Improved numerical dissipation for time integration algorithms in structural dynamics. Earthquake Engineering \& Structural Dynamics 5(3), 283-292 (1977)

19. Hughes, T.: The finite element method: linear static and dynamic finite element analysis. Dover Publications, New York (2000)

20. Hughes, T.J., Liu, W.: Implicit-explicit finite elements in transient analysis: I. stability theory; ii. implementation and numerical examples. J. Appl. Mech. 45, 371-378 (1978)

21. Hughes, T.J., Pister, K.S., Taylor, R.L.: Implicit-explicit finite elements in nonlinear transient analysis. Computer Methods in Applied Mechanics and Engineering 17, 159$182(1979)$

22. Hughes, T.J., Stephenson, R.A.: Convergence of implicit-explicit algorithms in nonlinear transient analysis. International Journal of Engineering Science 19(2), 295 - 302 (1981) 
23. Komatitsch, D., Liu, Q., Tromp, J., Süss, P., Stidham, C., Shaw, J.: Simulations of ground motion in the Los Angeles basin based upon the spectral-element method. Bulletin of the Seismological Society of America 94(1), 187-206 (2004)

24. Komatitsch, D., Tromp, J.: Introduction to the spectral element method for threedimensional seismic wave propagation. Geophysical Journal International 139(3), 806822 (1999)

25. Komatitsch, D., Tsuboi, S., Tromp, J.: The spectral-element method in seismology. In: Seismic Earth: Array Analysis of Broadband Seismograms, pp. 205-227. American Geophysical Union (2013)

26. Komatitsch, D., Vilotte, J.P.: The spectral element method: An efficient tool to simulate the seismic response of $2 \mathrm{~d}$ and $3 \mathrm{~d}$ geological structures. Bulletin of the Seismological Society of America 88, 368-392 (1998)

27. Lin, C.C., Lawton, E., Caliendo, J., Anderson, L.: An iterative finite element-boundary element algorithm. Computers \& Structures 59(5), 899-909 (1996)

28. Lysmer, J., Kuhlemeyer, R.L.: Finite-dynamic model for infinite media. Journal of Engineering Mechanics Division 95(4), 859-877 (1969)

29. Maday, Y., Patera, A.: Spectral element methods for the incompressible navier-stokes equations. In: State-of-the-art surveys on computational mechanics, pp. 71-143. New York (1989)

30. Mirhashemian, P., Khaji, N., Shakib, H.: Soil-structure interaction (SSI) analysis using a hybrid spectral element/finite element (SE/FE) approach. Journal of Seismology and Earthquake Engineering 11(2), 83-95 (2009)

31. Patera, A.: A spectral element method for fluid dynamics: laminar flow in a channel expansion. Journal of Computational Physics 54, 468-488 (1984)

32. Petersen, S., Dreyer, D., von Estorff O.: Assessment of finite and spectral element shape functions for efficient iterative simulations of interior acoustics. Computer Methods in Applied Mechanics and Engineering 195(44-47), 6463 - 6478 (2006)

33. Soares Jr, D.: An optimised FEM-BEM time-domain iterative coupling algorithm for dynamic analyses. Computers \& Structures 86(19-20), 1839-1844 (2008)

34. Soares Jr, D.: Coupled numerical methods to analyze interacting acoustic-dynamic models by multidomain decomposition techniques. Mathematical Problems in Engineering 2011, 28 (2011)

35. Soares Jr, D., von Estorff O., Mansur, W.J.: Efficient non-linear solid-fluid interaction analysis by an iterative BEM/FEM coupling. International Journal for Numerical Methods in Engineering 64(11), 1416-1431 (2005)

36. Soares Jr, D., Godinho, L.: An optimized BEM-FEM iterative coupling algorithm for acoustic-elastodynamic interaction analyses in the frequency domain. Computers \& Structures 106-107, 68-80 (2012)

37. Soares Jr, D., Rodrigues, G., Gonçalves, K.: An efficient multi-time-step implicitexplicit method to analyze solid-fluid coupled systems discretized by unconditionally stable time-domain finite element procedures. Computers \& Structures 88(5-6), $387-$ 394 (2010)

38. Szabo, B., Babuška, I.: Finite Element Analysis. Wiley (1991)

39. White, F.: Fluid Mechanics. McGraw-Hill Education (2015)

40. Wohlmuth, B.I.: A mortar finite element method using dual spaces for the lagrange multiplier. SIAM Journal on Numerical Analysis 38(3), 989-1012 (2000) 